\title{
NONLINEAR-COBWEB DYNAMICS IN THE APPROACH TO EQUILIBRIUM
}

\author{
J. M. GAFFNEY ${ }^{1}$ and C. E. M. PEARCE ${ }^{1}$
}

(Received 11 July, 2002)

\begin{abstract}
We discuss some propositions of Holmes and Manning relating to the evolution of price in a cobweb market approaching equilibrium. We find in particular that the detailed behaviour of the linear model is quite typical of nonlinear cobweb models.
\end{abstract}

\section{Introduction}

Recently Holmes and Manning [2] have given a treatment of the nonlinear cobweb model in which the usual assumption of a maximum price at which demand drops to zero is replaced by one of smoothness of the supply and demand curves together with a boundedness condition involving the ratio of their slopes. They gave a number of propositions describing the behaviour of this model when predicted prices are taken as the average of past actual prices. Proposition 1 says that invariable stability occurs as with the standard model of Carlson [1] and Manning [3]. Proposition 2 states that the convergence is eventually monotone.

Proposition 3 gives a detailed analysis for the linear cobweb. Here the market passes through a sequence of behaviour modes. Setting aside the special cases where the combination of initial price and market parameters is such that equilibrium is reached in a finite number of time periods, this is as follows. First there are undamped oscillations about equilibrium, then damped oscillations and finally a monotone convergence to equilibrium. The transitions between modes occur at times dependent on the ratio $\epsilon=\epsilon_{s} / \epsilon_{d}$ of the demand and supply elasticities at equilibrium.

Proposition 4 states that when a unimodality assumption is satisfied, onset of the last mode occurs at essentially the same time for a nonlinear cobweb. Finally, there

\footnotetext{
'School of Applied Mathematics, The University of Adelaide, SA 5005, Australia; e-mail: jgaffney@maths.adelaide.edu.au and cpearce@maths.adelaide.edu.au.

(C) Australian Mathematical Society 2004, Serial-fee code 1446-1811/04
} 
is a discussion of the parameter values that occur for actual markets. It is shown that convergence to equilibrium can be very slow and initially quite wild.

Our investigation centres on the ideas of Propositions 1 to 4 . We show in particular that the behaviour modes of the linear model as described by Holmes and Manning are generic for nonlinear models. Indeed, if a nonlinear market either starts sufficiently close to equilibrium or has the ratio $\epsilon$ sufficiently high, then not only does the market exhibit the same modes as in the linear case but the same onset times apply for transitions between those modes. These onset times then depend only on $\epsilon$.

\section{Discussion}

The analysis of Holmes and Manning is based on the properties of a nonautonomous difference system. Let $D$ denote the demand function and $S$ the supply function. These are taken as differentiable with $D^{\prime}<0$ and $S^{\prime}>0$. Then the realised price $P_{t}$ at time $t$ satisfies an equation

$$
P_{t}=D^{-1} \circ S\left[\frac{P_{t-1}}{t-1}+\frac{t-2}{t-1} S^{-1} \circ D\left(P_{t-1}\right)\right] \equiv f\left(P_{t-1}, t\right) .
$$

With $f_{1}=\partial f / \partial P_{t-1}$, Holmes and Manning derive the key equation

$$
f_{1}=\frac{S^{\prime}(\cdot)}{D^{\prime}(\cdot)} \frac{1}{t-1}+\frac{t-2}{t-1}
$$

on which Propositions 1-4 are based.

The suppressed arguments in this equation cause some difficulties in the analysis. To see this, consider an equation

$$
y=g^{-1} \circ w(x)
$$

in two differentiable and monotonic functions $g$ and $w$. This relation gives $g(y)=$ $w(x)$, whence $g^{\prime}(y) d y / d x=w^{\prime}(x)$ or

$$
\frac{d y}{d x}=\frac{w^{\prime}(x)}{g^{\prime}(y)}=\frac{w^{\prime}\left(w^{-1} \circ g(y)\right)}{g^{\prime}(y)} .
$$

With this paradigm we may apply the chain rule to (2.1) to derive

$$
f_{1}=\frac{S^{\prime}\left(S^{-1} \circ D\left(P_{t}\right)\right)}{D^{\prime}\left(P_{t}\right)}\left[\frac{1}{t-1}+\frac{t-2}{t-1} \frac{D^{\prime}\left(P_{t-1}\right)}{S^{\prime}\left(S^{-1} \circ D\left(P_{t-1}\right)\right)}\right] .
$$

Because the arguments of the first two occurrences of $S^{\prime}$ and $D^{\prime}$ are displaced in time from those of the second, it is not in general possible to reduce (2.4) to (2.2). However 
in the important special case of the linear cobweb $S^{\prime}$ and $D^{\prime}$ are both constants and (2.4) does reduce to (2.2). Hence Proposition 3, which relates to that case, is secure.

In this article we discuss Propositions 1 to 4 . To avoid the complications inherent in (2.1), we find it convenient to work in terms of an equivalent canonical recurrence relation involving the discrepancy $q_{t}=\widehat{P}_{t}-P^{e}$ between the predicted price $\widehat{P}_{t}$ applicable in period $t$ and the equilibrium price $P^{e}$.

The Carlson-Manning price-prediction rule is

$$
\widehat{P}_{t+1}=\left(1-\frac{1}{t}\right) \widehat{P}_{t}+\frac{1}{t} P_{t} .
$$

The assumption of market clearing gives

$$
P_{t}=D^{-1} \circ S\left(\widehat{P}_{t}\right),
$$

which enables us to express the price-prediction rule in canonical form as

$$
q_{t+1}=\left(1-\frac{1}{t}\right) q_{t}+\frac{1}{t} \phi\left(q_{t}\right)
$$

where

$$
\phi(q) \equiv D^{-1} \circ S\left(q+P^{e}\right)-P^{e} .
$$

Relation (2.5) encapsulates the dynamics of price evolution of the market. The function $\phi$ inherits the monotone-decreasing property of $D$, that is,

$$
\phi^{\prime}<0 \text {, }
$$

and since the demand and supply curves intersect at price $P^{e}$ we have

$$
\phi(0)=0 .
$$

The argument of Holmes and Manning is based on the assumption that $S^{\prime}(\cdot) / D^{\prime}(\cdot)$ is bounded. In our present notation this translates as $\phi^{\prime}$ bounded,

$$
\left|\phi^{\prime}\right| \leq K<\infty \text {, say. }
$$

In the following section we consider the evolution of the dynamical system given by (2.5) under the above-mentioned properties for $\phi$, which may be regarded as a relative elasticity function capturing the essential behaviour of the market. In fact we may treat (2.6) like (2.3) to derive

$$
\phi^{\prime}(0)=S^{\prime}\left(P^{e}\right) / D^{\prime}\left(P^{e}\right)=-\epsilon .
$$

We remark at the outset that it is sufficient to consider the price discrepancies $q_{t}$. The behaviour of the sequence $\left(q_{t}\right)$ about zero is that of the predicted-price sequence about $P^{e}$ which in turn via the well-known equivalence $P_{t} \gtrless P^{e}$ according as $\widehat{P}_{t} \lessgtr P^{e}$ mirrors the behaviour of the sequence of realised prices about equilibrium. 


\section{Results}

Relations (2.8) and (2.9) imply that $\phi$ must satisfy a Lipschitz condition

$$
|\phi(q)| \leq K|q| \text { for all } q \text {. }
$$

From this relation and (2.5), (2.7) and (2.8) we have that

$$
q_{t}<0 \text { implies } q_{t} \leq q_{t}(1-1 / t)<q_{t+1} \leq q_{t}[1-(1+K) / t]
$$

and

$$
q_{t}>0 \text { implies } q_{t} \geq q_{t}(1-1 / t)>q_{t+1} \geq q_{t}[1-(1+K) / t] .
$$

Therefore for all $t \geq T$, the smallest integer greater than or equal to $1+K$, the discrepancies $q_{t}, q_{t+1}$ are of the same sign or are both zero. Hence the sequence $\left(q_{t}\right)$ is eventually monotone. Also for $t \geq T$

$$
\left|q_{t+1}\right| \leq\left|q_{t}\right|(1-1 / t)
$$

and so

$$
\left|q_{t+1}\right| \leq\left|q_{T}\right| \prod_{r=T}^{t}(1-1 / r)=\left|q_{T}\right|(T-1) / t \rightarrow 0 \quad \text { as } t \rightarrow \infty .
$$

Thus $\left(q_{t}\right)$ eventually converges monotonically to limit zero and so $\left(P_{t}\right)$ eventually converges monotonically to the equilibrium price. This establishes Propositions 1 and 2.

At the conclusion of Proposition 2, Holmes and Manning state that the equilibrium price is approached from below. This does not seem to be necessarily so. For an example to the contrary, consider the linear cobweb model with $D^{\prime}=-2 S^{\prime}$. With these parameters $\phi(q)=-q / 2$ and

$$
q_{t+1}=\left(1-\frac{3}{2 t}\right) q_{t}
$$

Hence if the initial estimate $\widehat{P}_{1}$ of price is above $P^{e}$ then $q_{1}>0$ and $q_{t}<0$ for all $t>1$. Thus $\widehat{P}_{t}$ eventually approaches $P^{e}$ from below and so $P_{t}$ eventually approaches $P^{e}$ from above.

We now turn our attention to the time of the transition to eventual monotonicity. For particular initial prices, it can happen that the market achieves equilibrium in a finite number of periods. Setting aside such special values, we can argue as follows. When the market is near equilibrium, $q_{t}$ is small and since $\phi(0)=0$ and $\phi^{\prime}(0)=-\epsilon$ we have the expansion

$$
\phi\left(q_{t}\right)=-\epsilon q_{t}+\mathrm{O}\left(q_{t}^{2}\right)
$$


Substitution into (2.5) provides

$$
q_{t+1}=[1-(\epsilon+1) / n] q_{t}+\mathrm{O}\left(q_{t}^{2} / t\right)
$$

When $q_{t}^{2} / t$ is small we can conclude that predicted prices

(a) fluctuate with increasing amplitude about $P^{e}$ for $2 \leq t<(\epsilon+3) / 2$,

(b) fluctuate with decreasing amplitude about $P^{e}$ for $(\epsilon+3) / 2 \leq t<\epsilon+2$ and

(c) approach $P^{e}$ monotonically for $t \geq \epsilon+2$.

Realised prices must mirror this behaviour. This result provides a version of Proposition 3 for nonlinear cobweb markets and also frees Proposition 4 from its unimodality assumption. The analysis presupposes that the critical times are high enough that they are not passed before $q_{t}^{2} / t$ becomes sufficiently small for the approximations to be effective. This supposition will be the case when either the market starts near equilibrium or $\epsilon$ is large. Holmes and Manning adduced evidence that $\epsilon$ can be surprisingly large in some agricultural markets, that is, where the cobweb model is most likely to be applicable.

\section{References}

[1] J. A. Carlson, "An invariably stable cobweb model", Rev. Econ. Stud. 35 (1968) 360-362.

[2] J. M. Holmes and R. Manning, "Memory and market stability: the case of the cobweb", Econ. Lett. 28 (1988) 1-7.

[3] R. Manning, "A generalization of a cobweb theorem", Rev. Econ. Stud. 36 (1971) 123-125. 